\title{
Evaluación de la actividad insecticida de un producto granulado a base de Bacillus sphaericus sobre larvas de Anopheles albimanus y Culex quinquefasciatus en condiciones experimentales*
}

\author{
Estrella Cárdenas, Álvaro Rozo, Ligia Lugo**
}

Recibido: 10 de febrero de 2009 Revisado: 30 de abril de 2009 Aprobado: 18 de mayo de 2009

\section{Resumen}

Culex quinquefasciatus causa alergias por las picaduras y ha sido asociado con la encefalitis equina, mientras que Anopheles albimanus es vector de malaria. El objetivo fue evaluar la toxicidad de un producto granulado a base de Bacillus sphaericus sobre larvas de Culex quinquefasciatus y Anopheles albimanus. El estudio fue realizado sobre larvas de $C$. quinquefasciatus (Sibaté y Villavicencio) y An albimanus (Cartagena y Barranquilla). Las concentraciones ensayadas de B. sphaericus fueron: 20, 40, 60, 80, 100, 120, 140, 160, 180, 200 y 500 ppm para larvas de An. albimanus y para larvas de $C$. quinquefasciatus fueron: $2,4,6,8,10,12,14$ y 16 ppm. Se utilizaron 20 larvas en tres repeticiones por cada concentración del producto y el tiempo de exposición fue de 48 horas. El producto de B. sphaericus mostró alta mortalidad (entre 80 y 100\%) a bajas concentraciones (8 y $12 \mathrm{ppm}$ ) para larvas de C. quinquefasciatus; mientras que para larvas de An. albimanus a concentraciones más altas (entre 40 y 200 ppm) la mortalidad no superó el 50\%. La CL $\mathrm{L}_{50}$ Logit fue 176 ppm y la Probit fue 192 ppm de B. sphaericus para larvas de An. albimanus; mientras que para larvas de $C$. quinquefasciatus la $\mathrm{CL}_{50}$ Logit fue 1,9 ppm y la Probit fue 2, 2 ppm. Se concluye que las larvas de C. quinquefasciatus fueron más susceptibles a bajas concentraciones ( 2 a 12 ppm) de B. sphaericus, mientras que las larvas de An. albimanus mostraron alta toxicidad a concentraciones de $500 \mathrm{ppm}$.

\section{Palabras clave}

Bacillus sphaericus, Culex quinquefasciatus, Anopheles albimanus, mortalidad.

* Esta investigación fue financiada por la Universidad Santo Tomás, Universidad de La Salle y por el Instituto Nacional de Salud.

** Estrella Cárdenas, Universidad de La Salle, Bogotá; Álvaro Rozo, Universidad Santo Tomás, Bogotá; Ligia Lugo Universidad Santo Tomás, Bogotá, Grupo Entomología, Instituto Nacional de Salud, Bogotá, D.C. Correo electrónico: ecardenas@ lasalle.edu.co. 


\title{
Evaluation of insecticidal activity of a Bacillus sphaericus granulated product on Anopheles albimanus and Culex quinquefasciatus larvae in experimental conditions
}

\author{
Estrella Cárdenas, Álvaro Rozo, Ligia Lugo
}

\begin{abstract}
Culex quinquefasciatus causes allergies for the sting and has been associated with equine encephalitis disease; whereas, Anopheles albimanus is a vector of malaria. The objective was to evaluate the toxicity of a Bacillus sphaericus granulated product on Culex quinquefasciatus and Anopheles albimanus larvae. The study was realized on Culex. quinquefasciatus (Sibaté and Villavicencio) and Anopheles albimanus (Cartagena and Barranquilla) larvae. The tested concentrations of B. sphaericus were: 20, 40, 60, 80, 100, 120, 140, 160, 180, 200, 500 ppm for Anopheles albimanus larvae, and 2, 4, 6, 8, 10, 12, 14, $16 \mathrm{ppm}$ for $\mathrm{C}$. quinquefasciatus larvae. The exposure time was 48 hours, 20 larvae and three repetitions were used per concentration. The B. sphaericus product showed high mortality (between 80 and $100 \%$ ) to low concentrations (8-12ppm) for larvae of C. quinquefasciatus; whereas for An. Albimanus larvae to higher concentrations (between 40-200 ppm) mortality was minor 50\%. The Logit $\mathrm{LC}_{50}$ was 176 ppm and Probit was 192 ppm of B. sphaericus for An. albimanus larvae; whereas Logit $\mathrm{LC}_{50}$ was 1.9 ppm and Probit was 2.2 ppm for C. quinquefasciatus larvae. In conclusion C. quinquefasciatus larvae were susceptible to B. sphaericus product lower concentrations (2 and $12 \mathrm{ppm}$ ); whereas An. albimanus larvae showed high toxicity in 500 ppm of B. sphaericus product.
\end{abstract}

Key words

Bacillus sphaericus, Culex quinquefasciatus, Anopheles albimanus, mortality.
Recibido: 10 de febrero de 2009 Revisado: 30 de abril de 2009 Aprobado: 18 de mayo de 2009 


\section{INTRODUCCIÓN}

La especie Culex quinquefasciatus se encuentra en regiones tropicales y subtropicales con amplio rango de distribución (Savage \& Miller, 1995). Esta especie se ha relacionado con la transmisión de filarias (Wuchereria bancrofti y Dirofilaria immitis), del virus del Nilo occidental y de los virus causantes de la encefalitis de San Luis y la encefalitis Equina Venezolana (Salazar \& Moncada, 2004). Además del papel como vector de diferentes agentes patógenos, este mosquito provoca reacciones adversas, debidas a la saliva inoculada durante la picadura, las cuales generan manifestaciones clínicas generalizadas sobre sus hospederos como urticaria y angioedema, síntomas menos definidos como nauseas, dolor de cabeza y letargo (Travi \& Montoya, 1994; Peng \& Simons 1997). La severidad de la reacción depende de la sensibilidad individual y la gravedad de las reacciones locales está relacionada con el número de picaduras (Peng \& Simons 1997). Adicional a su acción hematófaga y vectorial, alta proliferación, actividad y densidad en áreas urbanas causan molestias sanitarias en el hombre y los animales; esto disminuye el bienestar y provoca una reducción en el trabajo, lo que ocasiona una desvalorización económica de las áreas y las propiedades.

En Colombia, C. quinquefasciatus se encuentra en la mayor parte del territorio nacional y es considerada una especie altamente antropofílica y está asociado con el hábitat humano en el área urbana y rural (Travi \& Montoya 1994); se encuentra en las regiones costeras, Llanos Orientales, las pluviselvas del Chocó y del Amazonas y en las laderas Andinas, y es la especie de mosquito más común en la ciudad de Bogotá, donde en algunas de sus áreas se constituye en un problema de salud pública, debido a la alergia ocasionada por su picadura y a las molestias causadas por las altas densidades de población que alcanzan, como ocurre en el municipio de Sibaté (Heinemann \& Belkin 1978).

Algunas especies del género Anopheles son vectores de malaria; la cual es la enfermedad más importante transmitida por insectos. En Colombia se reporta la presencia de aproximadamente 45 especies de Anopheles, de las cuales son consideradas vectores de malaria, las siguientes: Anopheles darlingi, An. albimanus y An. nuneztovari (Olano et al., 2001). En nuestro país, la malaria es un grave problema de salud pública, debido a que cerca del $85 \%$ del territorio rural colombiano, situado por debajo de los $1.600 \mathrm{msnm}$, presenta condiciones climáticas, geográficas y epidemiológicas aptas para la transmisión de la enfermedad. Se estima que cerca de 25 millones de personas se encuentran en riesgo de enfermar o morir por esta causa. A finales de 2005 se presentaron en Colombia un total de 107.866 casos de malaria (INS, 2005), para el año 2006 hasta la semana epidemiológica 46 se presentaron un total de 71.883 casos nuevos de malaria. El grupo de edad más afectado estuvo entre quince y cuarenta y cuatro años. Los departamentos que mostraron mayor número de casos fueron: Antioquia, Nariño, Córdoba, Choco, Meta, Guaviare y Putumayo (SIVIGILA, 2005).

El uso de insecticidas organosintéticos para el control de insectos vectores es afectado por el desarrollo de resistencia en las poblaciones naturales de mosquitos, lo cual se refleja directamente en la reemergencia de enfermedades transmitidas por insectos 
(Brogdon \& MacAllister, 1998). El control biológico se considera como una alternativa para minimizar las poblaciones de insectos vectores; consiste en la utilización de agente biológico con capacidad para reducir el tamaño de la una población de vectores o plagas. Tal agente puede ser un depredador, un patógeno, un competidor o una toxina derivada de plantas o de microorganismos (Vargas, 1998).

\section{Bacterias con propiedades insecticidas}

Se destacan Bacillus thurigiensis, Bacillus sphaericus y Bacillus latesrosporus como las bacterias más promisorias como biocontroladores de mosquitos. B. sphaericus es altamente tóxica para larvas de los géneros Anopheles y Culex (Berti et al., 2002). La bacteria Bacillus sphaericus es altamente toxica para larvas del género Anopheles (Murat, 1995, Rivera, 1995; Blanco et al., 2000). B. sphaericus es efectivo contra especies de Culex y Anopheles y menos efectivo en Aedes; mientras que $B$. thuringiensis subsp. israelensis es efectivo contra especies de Aedes y Culex y menos efectivo contra especies de Anopheles (Davidson et al., 1984). Adicionalmente, B. sphaericus tiene actividad toxica y alta persistencia en aguas contaminadas en las cuales la toxicidad de $B$. thuringiensis subsp. israelensis se pierde rápidamente (Lacey \& Undeen 1986). Las preparaciones particulares de estas bacterias que contienen igual cantidad de toxina; pueden diferir con respecto al tamaño del granulado del producto comercial (Baumann et al., 1991).

\section{Materiales y MÉtodos}

Se emplearon larvas de las colonias de $A n$. Albimanus procedentes de Barranquilla (de- partamento del Atlántico) y Cartagena (Bolívar); fueron mantenidas en el Laboratorio de Entomología del Instituto Nacional de Salud. Se recolectaron adultos y larvas de $C$. quinquefasciatus procedentes de Villavicencio (Meta) y del Embalse del Muña en Sibaté (Cundinamarca).

Para el mantenimiento de las colonias de insectos se utilizó el insectario cuyas condiciones ambientales fueron las siguientes: temperatura $28+-2^{\circ} \mathrm{C}$, humedad relativa de $62+-5 \%$ y fotoperiodo de 12 horas luz y 12 horas oscuridad.

Las larvas se mantuvieron en bandejas plásticas (ancho $22 \mathrm{~cm}$, largo $22 \mathrm{~cm}$ y alto $10 \mathrm{~cm}$ ), fueron alimentadas con rodentina ${ }^{\circledR}$ pulverizada. Los adultos se mantuvieron en jaulas entomológicas; para la alimentación de las hembras con sangre se procedió a colocarles dentro de la jaula cada tercer día un ratón anestesiado por una hora, se suministró glucosa al 30\% en motas de algodón; además se proporcionó una vasija de plástico con agua sin cloro para la oviposición. Se utilizaron larvas del IV instar de ambas especies para los bioensayos. Para la determinación taxonómica de los mosquitos de Villavicencio, se realizó siguiendo las claves de Cova-García et al. (1996).

\section{Bioensayos con el producto larvicida Bacillus sphaericus}

Los ensayos se realizaron en el Laboratorio de Entomología del Instituto Nacional de Salud. Bacillus sphaericus 2362 es el ingrediente activo del biolarvicida comercial y el producto contiene $51,2 \%$ de ingrediente activo y $48,8 \%$ de ingredientes inertes. Con base en esta información se preparó un litro de solución stock de 500 ppm y de cual 
se realizaron las diluciones para los bioensayos. Se probaron varias concentraciones; para larvas del cuarto estadio de C. quinquefasciatus se ensayaron las siguientes concentraciones del producto: 2, 4, 6, 8, 10, 12, 14 y 16 ppm y para larvas del cuarto estadio de An. albimanus se ensayaron las siguientes concentraciones: 20, 40, 60, 80, 100, 120, 140, 160, 180, 200 y 500 ppm. Las larvas tratadas se revisaron a las 24 y 48 horas y se registró el número de larvas muertas, para determinar el porcentaje de mortalidad causada por el biolarvicida.

\section{Análisis estadístico}

Se utilizó un diseño experimental completamente al azar consistente en tres réplicas con 20 larvas cada una y tres repeticiones del tratamiento y un control; se analizaron los datos por Probit y Logit (McCullagh \& Nelder 1989; Cox \& Hinkley, 1974; Hosmer \& Lemeshow, 1989). Para la evaluación de la significancia estadística de los parámetros del modelo se utilizó la prueba estadística test de Wald (Cox \& Hinkley, 1974). Para la determinación de la concentración letal 50, CL50 y la concentración letal 95, CL95, así como sus respectivos intervalos para un nivel de confianza del 95\% usó la prueba estadística de Hosmer y Lemeshow (1989).

\section{Consideraciones éticas}

Este proyecto no contempló procedimientos en humanos, por el contrario, los resultados contribuyen al mejoramiento de la calidad de vida de las comunidades de Sibaté y Vi- llavicencio (Barrio La Casona). Se propusieron métodos alternativos al uso de insecticidas químicos. Se utilizaron ratones de laboratorio de la colonia ICR del INS para el mantenimiento de las colonias de insectos colectados en el campo, los ratones anestesiados con pentotal se pusieron en las jaulas de mosquitos por 30 minutos/día, cada tercer día. Los animales fueron manipulados en concordancia con las regulaciones éticas nacionales e internacionales vigentes (Ministerio de Salud, 1993, NRCC 2002).

\section{Resultados}

\section{Mortalidad en larvas de}

\section{Anopheles albimanus, ocasionada por el biolarvicida Bacillus \\ sphaericus}

La tabla 1 muestra que el porcentaje de mortalidad en larvas de cuarto instar de las dos colonias An. albimanus por efecto de diez concentraciones en partes por millón del producto fue baja a las 24 horas; mientras que a las 48 horas la mortalidad se incrementó; no obstante, estuvo por debajo del $50 \%$ en las concentraciones de 20 a 200 ppm. La concentración 500 ppm mostró más efectividad a las 48 horas; lo cual sugiere que se deben ensayar concentraciones entre 220 y 480 ppm para determinar la concentración letal 50 para esta especie en condiciones de laboratorio. En el control no se observó mortalidad de larvas a las 24 horas ni a las 48 horas. 
Tabla 1. Porcentaje de mortalidad de larvas de Anopheles albimanus (colonia Barranquilla y colonia Cartagena) a las 24 y 48 horas después de la aplicación de diez concentraciones en partes por millón de Bacillus sphaericus 2362. Para ensayar cada concentración se utilizaron 60 larvas

\begin{tabular}{|c|c|c|c|c|}
\hline \multirow{2}{*}{$\begin{array}{c}\text { Concentraciones } \\
\text { en ppm }\end{array}$} & \multicolumn{2}{|c|}{$\%$ larvas muertas a las 24 horas } & \multicolumn{2}{|c|}{$\%$ larvas muertas a las 48 horas } \\
\hline & $\begin{array}{c}\text { Anopheles } \\
\text { Barranquilla }\end{array}$ & $\begin{array}{l}\text { Anopheles } \\
\text { Cartagena }\end{array}$ & $\begin{array}{c}\text { Anopheles } \\
\text { Barranquilla }\end{array}$ & $\begin{array}{l}\text { Anopheles } \\
\text { Cartagena }\end{array}$ \\
\hline 20 & 1,7 & 3,3 & 16,7 & 15,0 \\
\hline 40 & 3,3 & 0,0 & 33,3 & 28,3 \\
\hline 60 & 5,0 & 6,7 & 36,7 & 36,7 \\
\hline 80 & 3,3 & 1,7 & 36,7 & 36,7 \\
\hline 100 & 3,3 & 0,0 & 35,0 & 25,0 \\
\hline 120 & 11,7 & 3,3 & 33,3 & 28,3 \\
\hline 140 & 0,0 & 1,7 & 40,0 & 35,0 \\
\hline 160 & 3,3 & 6,7 & 36,7 & 33,3 \\
\hline 180 & 6,7 & 6,7 & 46,7 & 45,0 \\
\hline 200 & 5,0 & 0,0 & 48,3 & 43,3 \\
\hline 500 & 86,7 & 90,0 & 100,0 & 100,0 \\
\hline Control & 0 & 0 & 0 & 0 \\
\hline
\end{tabular}

La tabla 2 presenta los estimativos de los parámetros de los modelos Probit y Logit con su respectivo error estándar y test de significancia. El error estándar es una medida de la precisión de la estimación, en la cual valores grandes de esta medida indican alta variabilidad en los datos. El test de significancia permite establecer el efecto de las variables (estímulos) sobre la respuesta de interés. Por ejemplo, el test de significancia y el P-valor para $\beta_{1}$ permiten establecer si el biolarvicida Bacillus sphaericus tuvo efecto sobre la tasa de mortalidad de las larvas de los mosquitos. Valores de P-valor menores al 0,05 indican que Bacillus sphaericus tuvo efecto estadísticamente significativo sobre la mortalidad de las larvas de Anophleles albimanus de las colonia de Barranquilla y Cartagena.

Tabla 2. Estimación de los parámetros para los modelos en la mortalidad en larvas de Anopheles albimanus, ocasionada por el biolarvicida Bacillus sphaericus en concentraciones: 20, 40, 60, 80, 100, 120, 140, 160, 180,

200 y 500 ppm; después de 48 horas de la aplicación

\begin{tabular}{|l|l|l|l|l|l|}
\hline \multirow{2}{*}{ Modelo } & \multicolumn{5}{|l|}{ Colonia Barranquilla } \\
\cline { 2 - 6 } & Parámetro & Estimativa & Error Estándar & Test & P-valor \\
\hline \multirow{2}{*}{ Logit } & $\beta_{0}$ & $-1,737$ & 0,156 & $-11,157$ & $<0,001$ \\
\cline { 2 - 6 } & $\beta_{1}$ & 0,009 & 0,001 & 8,984 & $<0,001$ \\
\hline \multirow{2}{*}{ Probit } & $\beta_{0}$ & $-1,060$ & 0,087 & $-12,120$ & $<0,001$ \\
\cline { 2 - 6 } & $\beta_{1}$ & 0,006 & 0,001 & 9,890 & $<0,001$ \\
\hline \multirow{3}{*}{ Logit } & Colonia Cartagena & $-1,906$ & 0,159 & $-1,196$ & $<0,001$ \\
\cline { 2 - 6 } & $\beta_{0}$ & 0,009 & 0,001 & 9,099 & $<0,001$ \\
\hline \multirow{2}{*}{ Probit } & $\beta_{0}$ & $-1,156$ & 0,088 & $-13,130$ & $<0,001$ \\
\cline { 2 - 6 } & $\beta_{1}$ & 0,006 & 0,001 & 10,140 & $<0,001$ \\
\hline
\end{tabular}


A continuación se presentan los valores del estadístico de Hosmer y Lemeshow (1989) para los modelos Probit y Logit sobre la mortalidad de larvas de Anopheles albimanus de las colonias de Barranquilla y Cartagena ocasionada por la bacteria B. sphaericus. Probit (test=29,32; $\mathrm{P}=<0,001$ ) y Logit (test=30,76; $\mathrm{P}=<0,001)$ para la colonia de Barranquilla; mientras que para la colonia de Cartagena los valores fueron Probit (test $=32,72$; $\mathrm{P}=<0,001)$ y Logit (test $=33,94 ; \mathrm{P}=<0,001)$. Este test permite establecer si el modelo estimado describe adecuadamente el conjunto de datos. Valores de P-valor mayores a 0,05 indican que el modelo ajustado describe adecuadamente el conjunto de observaciones. Para el caso los valores de P-valor fueron menores a 0,05 ; posiblemente, debido a la variación en la tasa de mortalidad a medida que se varía la concentración; es decir, en la medida en que se aumentaba la concentración no siempre aumentaba la mortalidad que era lo esperado.

\section{Estimación de la CL50 y de CL95 de VectoLex (Bacillus sphaericus) sobre larvas de Anophleles albimanus}

La tabla 3 presenta la estimación de las concentraciones letales con sus respectivos intervalos de confianza (al 95\%) calculados bajo los dos modelos propuestos. Para el límite inferior se usa la abreviación $L i$ mientras que para el límite superior se usa la abreviación Ls.

Tabla 3. Concentraciones letales CL50 y CL95, intervalos de confianza para los modelos Logit y Probit en la mortalidad de larvas de Anopheles albimanus, ocasionada por el biolarvicida Bacillus sphaericus en concentraciones: 20, 40, 60, 80, 100, 120, 140, 160, 180, 200 y 500 ppm; después de 48 horas

\begin{tabular}{|l|l|l|l|l|}
\hline \multirow{2}{*}{ Concentraciones } & \multicolumn{5}{|c|}{ Colonia Barranquilla } \\
\cline { 2 - 5 } & \multicolumn{1}{|c|}{ Modelo } & \multicolumn{1}{|c|}{ DL } & \multicolumn{1}{c|}{ L. I } & \multicolumn{1}{c|}{ L. S } \\
\hline \multirow{3}{*}{ CL50 } & Logit & 176,60 & 158,87 & 194,32 \\
\cline { 2 - 6 } & Probit & 176,64 & 159,42 & 193,85 \\
\hline \multirow{3}{*}{ CL95 } & Logit & 475,95 & 409,06 & 542,83 \\
\cline { 2 - 6 } & Probit & 450,56 & 393,33 & 507,86 \\
\hline \multirow{3}{*}{ CL50 } & Colonia Cartagena & 192,88 & 173,60 & 212,16 \\
\hline \multirow{2}{*}{ CL95 } & Logit & 192,73 & 174,25 & 211,21 \\
\cline { 2 - 5 } & Probit & 490,82 & 422,72 & 558,92 \\
\cline { 2 - 5 } & Logit & 466,76 & 408,79 & 524,74 \\
\cline { 2 - 5 } & Probit & & \\
\hline
\end{tabular}




\section{Mortalidad en larvas de Culex}

quinquefasciatus, ocasionada

por el biolarvicida Bacillus

\section{sphaericus}

La tabla 4 muestra que el porcentaje de mortalidad en larvas de cuarto instar de las dos colonias C. quinquefasciatus por efecto de ocho concentraciones en partes por millón de Vectolex estuvo entre 18,3 y $80 \%$; a las 24 horas; mientras que a las 48 horas la mortalidad estuvo entre el 73,3 y el 100\%. Como se observa esta especie es más susceptible a bajas concentraciones de VectoLex, comparado con Anopheles albimanus. Los controles no mostraron mortalidad de larvas a las 24 ni a las 48 horas

Tabla 4. Porcentaje de mortalidad de larvas de Culex quinquefasciatus (colonia Sibaté y colonia Villavicencio) a las 24 y 48 horas después de la aplicación de ocho concentraciones en partes por millón de Bacillus sphaericus 2.362. Para ensayar cada concentración se utilizaron 60 larvas

\begin{tabular}{|c|c|c|c|c|}
\hline \multirow{2}{*}{$\begin{array}{c}\text { Concentraciones } \\
\text { en ppm }\end{array}$} & \multicolumn{2}{|c|}{ \% larvas muertas 24 horas } & \multicolumn{2}{c|}{ \% larvas muertas a las 48 horas } \\
\cline { 2 - 5 } & Culex Sibate & $\begin{array}{c}\text { Culex } \\
\text { Villavicencio }\end{array}$ & Culex Sibate & $\begin{array}{c}\text { Culex } \\
\text { Villavicencio }\end{array}$ \\
\hline 2 & 18,3 & 20,0 & 76,7 & 73,3 \\
\hline 4 & 33,3 & 31,7 & 93,3 & 86,7 \\
\hline 6 & 28,3 & 31,7 & 80,0 & 81,7 \\
\hline 8 & 73,3 & 76,7 & 96,7 & 93,3 \\
\hline 10 & 70,0 & 63,3 & 100,0 & 98,3 \\
\hline 12 & 81,7 & 88,3 & 100,0 & 100,0 \\
\hline 14 & 65,0 & 70,0 & 100,0 & 100,0 \\
\hline 16 & 80,0 & 70,0 & 100,0 & 100,0 \\
\hline Control & 0 & 0 & 0 & 0 \\
\hline
\end{tabular}

La tabla 5 presenta los estimativos de los parámetros de los modelos Probit y Logit con su respectivo error estándar y test de significancia en la mortalidad en larvas Culex quinquefasciatus, ocasionada por el biolarvicida Bacillus sphaericus. Valores de
P-valor menores al 0,05 indican que Bacillus sphaericus tuvo efecto estadísticamente significativo sobre la mortalidad de las larvas de Culex quinquefasciatus de las colonias de Sibaté y Villavicencio.

Tabla 5. Estimación de los parámetros para los modelos en la mortalidad de larvas de Culex quinquefasciatus, ocasionada por el biolarvicida Bacillus sphaericus en 2, 4, 6, 8, 10, 12, 14, y 16 ppm; después de 48 horas de la aplicación

\begin{tabular}{|c|c|c|c|c|c|}
\hline \multirow{2}{*}{ Modelo } & \multicolumn{5}{|c|}{ Colonia Sibaté } \\
\cline { 2 - 6 } & Parámetro & Estimativa & Error Estándar & Test & P-valor \\
\hline \multirow{2}{*}{ Logit } & $\beta_{0}$ & $-1,328$ & 0,250 & -5308 & $<0,001$ \\
\cline { 2 - 6 } & $\beta_{1}$ & 0,698 & 0,077 & 9,040 & $<0,001$ \\
\hline \multirow{2}{*}{ Probit } & $\beta_{0}$ & 0,743 & 0,141 & $-5,249$ & $<0,001$ \\
\cline { 2 - 6 } & $\beta_{1}$ & 0,370 & 0,038 & 9,701 & $<0,001$ \\
\hline & $\beta_{0}$ & $-1,279$ & 0,237 & $-5,395$ & $<0,001$ \\
\hline \multirow{2}{*}{ Logit } & $\beta_{1}$ & 0,605 & 0,064 & 9,456 & $<0,001$ \\
\cline { 2 - 6 } & $\beta_{0}$ & $-0,175$ & 0,135 & $-5,283$ & $<0,001$ \\
\hline \multirow{2}{*}{ Probit } & $\beta_{1}$ & 0,324 & 0,032 & 10,096 & $<0,001$ \\
\cline { 2 - 6 }
\end{tabular}


Los valores del estadístico de Hosmer y Lemeshow (1989) para los modelos Probit y Logit sobre la mortalidad de larvas de $\mathrm{Cu}$ lex quinquefasciatus de las colonias de Sibate (Cundinamarca) y Villavicencio (Meta) ocasionada por la bacteria B. sphaericus fueron los siguientes: Probit (test $=61,74 ; \mathrm{P}=<0,001$ ) y Logit (test $=63,56 ; \mathrm{P}=<0,001$ ) para la colonia de Sibaté; mientras que para la colonia de Villavicencio los valores fueron Probit (test=48,76; $\mathrm{P}=<0,001$ ) y Logit (test $=46,84$; $\mathrm{P}=<0,001)$. Valores de $\mathrm{P}$-valor mayores a 0,05 indican que el modelo ajustado describe adecuadamente el conjunto de observaciones. Para el caso los valores de P-valor fueron menores a 0,05 ; posiblemente, debido a la variación en la tasa de mortalidad a medida que se varía la concentración, es decir, en la medida en que se aumentaba la concentración no siempre aumentaba la mortalidad, que era lo esperado.

\section{Estimación de la CL50 y de CL95 de VectoLex (Bacillus sphaericus) sobre larvas de Culex quinquefasciatus}

La tabla 6 presenta la estimación de las concentraciones letales con sus respectivos intervalos de confianza (al 95\%) calculados bajo los dos modelos propuestos. Para el límite inferior se usa la abreviación $L i$, mientras que para el límite superior se usa la abreviación Ls.

Tabla 6. Concentraciones letales CL50 y CL95, intervalos de confianza bajo los modelos Logit y Probit en la mortalidad de larvas de Culex quinquefasciatus, ocasionada por el biolarvicida Bacillus sphaericus en 2, 4, $6,8,10,12,14$, y 16 ppm; después de 48 horas de la aplicación

\begin{tabular}{|c|c|c|c|c|}
\hline \multirow{2}{*}{ Concentraciones } & \multicolumn{4}{|c|}{ Colonia Sibaté } \\
\cline { 2 - 5 } & Modelo & DL & L. I & L. S \\
\hline \multirow{2}{*}{ CL50 } & Logit & 1,901 & 1,506 & 2,295 \\
\cline { 2 - 5 } & Probit & 2,001 & 1,582 & 2,433 \\
\hline \multirow{2}{*}{ CL95 } & Logit & 6,116 & 5,351 & 6,881 \\
\cline { 2 - 5 } & Probit & 6,449 & 5,722 & 7,176 \\
\hline \multirow{2}{*}{ CL50 } & Logit & 2,111 & 1,679 & 2,543 \\
\cline { 2 - 5 } & Probit & 2,206 & 1,740 & 2,672 \\
\hline \multirow{2}{*}{ CL95 } & Logit & 6,971 & 6,142 & 7,805 \\
\cline { 2 - 5 } & Probit & 7,279 & 6,498 & 8,062 \\
\hline
\end{tabular}

\section{Discusión}

La actividad toxica de Bacillus sphaericus sobre larvas de mosquitos ha sido ampliamente estudiada en el mundo (Arredondo et al., 1990; Baumann et al., 1991; Berry et al., 1993; Consoli et al., 1997; Castro et al., 1996; Des Roches \& Garcfa, 1984; Jones et al., 1990; La- cey et al., 1986; Majori et al., 1987; Mian \& Mulla, 1983; Montero et al., 1991; Mulla et al., 1991; Nicolas \& Dossou, 1987; WHO, 1985; Yousten et al., 1982); y en Colombia se han realizado varios estudios sobre actividad toxica de Griselesf en formulación líquida de B. sphaericus en larvas de mosquitos Culex quinquefasciatus, Aedes taeniorhynchus y Anopheles 
albimanus en Buenaventura en condiciones de campo (Villarreal, 1995), y en sumideros de Cali se utilizaron productos químicos y VectoMax para el control de larvas de mosquitos (Perez et al., 2008). En Medellín se realizaron estudios para la introducción de genes de $B$ thuringiensis dentro de $B$. sphaericus para aumentar la efectividad de esta última contra larvas de mosquitos (Thiery et al., 1998); sin embargo, bioensayos en Colombia, con formulación granulada de B. sphaericus para larvas de mosquitos, han sido poco explorados.

El presente estudio analizó la actividad larvicida de un producto comercial a base de B. sphaericus en concentraciones de partes por millón sobre larvas de An. albimanus y C. quinquefasciatus, el cual mostró alta toxicidad a bajas concentraciones (8 a $12 \mathrm{ppm}$ ) para larvas de $C$. quinquefasciatus; resultados acordes con lo reportado por Zahiri et al. (2004) en condiciones de laboratorio realizado en la Universidad de California. Para larvas de An. albimanus en concentraciones entre 40 y 200 ppm el porcentaje de mortalidad fue menor del $50 \%$. La $\mathrm{CL}_{50}$ Logit del extracto acuoso de $R$. graveolens estuvo entre 96 y $138 \mathrm{mg} / \mathrm{L}$; mientras que la $\mathrm{CL}_{50}$ Probit estuvo entre 109, 7 y 143, 7 mg/L para larvas de An. albimanus; mientras que para larvas de C. quinquefasciatus la $\mathrm{CL}_{50}$ Logit estuvo entre 135 y 203 mg/L y la Probit estuvo entre 148 y 109 mg/L. La CL ${ }_{50}$ Logit y Probit de B. sphaericus estuvo entre 176 y 192 ppm para larvas de An. albimanus; mientras que para larvas de $C$. quinquefasciatus la $\mathrm{CL}_{50}$ Logit estuvo entre 1, 9 y 2, 1 ppm y la Probit entre 2 y 2,2 ppm.

Se concluye que el extracto acuoso de $R$. graveolens en concentración de $300 \mathrm{mg} / \mathrm{L}$ mostró alta toxicidad tanto para larvas de An. albimanus como para larvas de C. quinquefasciatus. Las larvas de C. quinquefasciatus fueron más susceptibles a concentraciones bajas (8 a $12 \mathrm{ppm}$ ) de B. sphaericus comparadas con las larvas de An. albimanus que sólo mostraron alta toxicidad a concentraciones de 500 ppm.

\section{Agradecimientos}

A la Universidad de La Salle, a la Universidad Santo Tomás y al Instituto Nacional de Salud por la financiación de la investigación. Al Laboratorio de Entomología del Instituto Nacional de Salud, por permitirnos trabajar con las colonias de referencia de Anopheles albimanus y por la colaboración en el mantenimiento de las colonias de $\mathrm{Cu}$ lex quinquefasciatus. A la Dra. Luz Marina Rondón Poveda, MSc. en Estadística; por su asesoría en la parte estadística de la investigación. Al auxiliar de laboratorio John Muñoz, del Instituto Nacional de Salud; por su colaboración en el mantenimiento de las colonias y suministro de las larvas en los tiempos establecidos. 


\section{REFERENCIAS}

Arredondo, A., López, T., Rodríguez, M. \& Bown, D. (1990). Small Scale Field Trials of Bacillus sphaericus (strain 2362) against Anopheline and Culicine Mosquito Larvae in Southern Mexico. Journal American Mosquitoes Control Association, 6 (2), 300-305.

Baumann, P., Clark, M., Baumann, L. \& Broadwell A. (1991). Bacillus sphaericus as a Mosquito Pathogen: Properties of the Organism and its Toxin. Microbiology Reviews, 55, 425-436.

Baumann, P., Clark, M.A., Baumann, L. \& Broadwell, A.H. (1991). Bacillus sphaericus as a Mosquito Pathogen: Properties of the Organism and Its Toxins. Microbiological Reviews, 55 (3), 425-436.

Berry, C., Hindley, J., Ehrhardt, A., Grounds, T., Souza, L. \& Davidson, E. (1993). Genetic Determinants of the Host Range of the Bacillus Sphaericus Mosquito Larvicidal Toxins. Journal Bacteriology, 175, 510-518.

Berti, J., Ramírez, X., Gonzáles, J.E. \& Herrera, M. Evaluación de la efectividad de Bacillus sphaericus contra Anopheles aquasalis Curry (Diptera: Culicidae) en curaderos naturales del Estado Sucre, Venezuela. Entomotropica, 17 (1), 1-5.

Blanco, S.D., Martínez, A., Cano, O.R., Tello, R. \& Mendoza, I. (2000). Introducción del Bacillus sphaericus cepa-2362 (GRISELESF) para el control biológico de vectores maláricos en Guatemala. Rev Cubana Med Trop, 52 (1), 37-43.

Brogdon, W.G. \& McAllister, J.C. Insecticide Resistance and Vector Control. Emerging Infectious Diseases, 4 (4), 605-613.
Castro, J., García, L. \& Neyra, D. Evaluación del tratamiento con Bacillus sphaericus 2362 en criaderos naturales en zonas de alto riesgo de malaria. Revista Peruana de Epidemiología, 9 (2), 18-23.

Consoli, R., De Santos, B., Lamounier, M., Secundino, N., Rabinovitch, L., Silva, C., Alves, R. \& Carneiro, N. (1997). Efficacy of a New Formulation of Bacillus sphaericus 2362 against Culex quinquefasciatus (Diptera: Culicidae) in Montes Claros, Minas Gerais, Brazil. Memorias do Instituto Oswaldo Cruz, Río de Janeiro, 92 (4), 571-573.

Cova-García, P., Sutil, E. \& Rasseau, J. (1996). Mosquitos (culicinos) de Venezuela. Caracas, Venezuela: Publicaciones del Ministerio de sanidad y Asistencia Social.

Cox, D.R. \& Hinkley, D.V. (1974). Theorical Statistics. London: Chapman and Hall.

Davidson, E.W., Urbina, M., Payne, J., Mulla, M.S., Darwazeh, H., Dulmage, H.T. \& Correa, J.A. (1984). Fate of Bacillus sphaericus 1593 and 2362 spores used as larvicides in the aquatic environment. Appl. Environ. Microbiol, 47, 125-129.

Des Rochers, B. \& Garcfa, R. Evidence for Persistence and Recycling of Bacillus sphaericus. Mosquito News, 44 (2), 160165.

Heinemann, S.J. \& Belkin, J.N. (1978). Collection Records of the Project "Mosquitoes of Middle America" 12, Colombia (COA, COB, COL, COM). Mosq Syst, 10, 493-539.

Hosmer, D.W. \& Lemeshow, S. (1989). Applied Logistic Regression. New York: John Wiley. 
INS. (2005). Informe Final Malaria semanas. Colombia: Instituto Nacional de Salud.

Jones, J., Weathersbee, A., Efird, P. \& Meisch, M. (1990). Evaluation of Bacillus sphaericus 2362 against Culex quinquefasciatus in Septic Ditches. Journal American Mosquitoes Control Association, 6 (3), 496-499.

Lacey, L., Heitzman, C., Meisch, M. \& Biliodeaux, J. (1986). Becomist Applied Bacillus sphaericus for the Control of Riceland Mosquitoes. Journal American Mosquitoes Control Association, 2 (4), 548-551.

Lacey, L.A. \& Undeen, A.H. (1986). Microbial Control of Black Flies and Mosquitoes. Annu. Rev. Entomol, 31, 265-296.

Majori, G., Ali, A. \& Sabatinelli, G. (1987). Laboratory and Field Efficacy o Bacillus Thuringiensis Var. Isra Elensis and Bacillus sphaericus against Anopheles gambiae S. L. and Culex quinquefasciatus in Ouagadougou. Burkina Faso. Journal American Mosquitoes Control Association, 3 (1), 20-24.

McCullagh, P. \& Nelder, J.A.(1989). Generalized Linear Models. Monographs on Statistics and Applied Probability (Vol. 37, $2^{\text {nd }}$ edition). London: Chapman and Hall.

Mian, L.S. \& Mulla, M.S. (1983). Factors Influencing Activity of the Microbial Agent Bacillus sphaericus against Mosquito Larvae. Bulletin Society Vector Eco$\log y, 8,128-134$.

Montero, G., Díaz, M., Marrero, A. \& Casti1lo A. (1991). Resultados de las aplicaciones en pilotaje del biolarvicida Bacillus sphaericus 2362 en criaderos de mosquitos del municipio Santa Cruz del Norte (Provincia La Habana). Revista Cubana de Medicina Tropical, 43 (1), 39-44.
Mulla, M.S., Singh, N. \& Darwazeh, H.A. (1991). Delayed Mortality and Morphogenetic Anomalies Induced in Culex quinquefasciatus by the Microbial control Agent Bacillus Sphaericus. Journal American Mosquitoes Control Association, 7 (3), 412-419.

Murat E., Y. (1995). Bacillus sphaericus 2362 Calcium Alginate Microcapsules for Mosquitoes Control. Enzyme Microbiol Technol, 17, 587-591.

Nicolas, L. \& Dossou-Yovo, J. (1987). Differential Effects of Bacillus sphaericus Strain 2362 on Culex Quinquefasciatus and its Competitor Culex cinereus in West Africa. Medical and Veterinary Entomology, 1, 23-27.

NRCC. (2002). Guía para el cuidado y uso de los animales de Laboratorio. Institute of Laboratory Animal Resources Commission on Life Sciences. México: National Research Council of Canada. Versión en Español. Edición Mexicana auspiciada por la Academia Nacional de Medicina.

Olano, V., Brochero, H., Sáenz, R., Quiñones, M. \& Molina, J. Mapas preliminares de la distribución de Anopheles vectores de malaria en Colombia. Biomédica, 21 (4), 402-403.

Peng, Z. \& Simons, E. (1997). Cross-Reactivity of Skin and Serum Specific IgE Responses and Allergen Analysis for Three Mosquito Species with Worldwide Distribution. J Allergy Clin Immunol, 100, 192-8. 23.

Pérez, M., Ocampo, C., Giraldo-Calderón, G.I., Morales, C. (2008). Evaluación del triflumuron y la mezcla de Bacillus thuringiensis mas Bacillus sphaericus para el control de las formas imnaduras de Aegypti y Culex quinquefasciatus en sumideros en Cali, Colombia. Biomédica, 28 (2), 224-233. 
República de Colombia, Ministerio de Salud. Resolución № 008430 de 1993. Por la cual se establecen las normas científicas, técnicas y administrativas para la investigación en salud.

Rivera, P. (1997). Evaluación de la efectividad biolarvicida y residual de Bacillus sphaericus (cepa 2362) para el control de Anopheles albimanus en la costa del lago Xolotlan, Managua, Nicaragua, 1995. Rev Nicar Entomol, 42, 7-14.

Salazar, M.J. \& Moncada, L.I. (2004). Ciclo de vida de Culex quinquefasciatus Say, 1826 (Diptera: Culicidae) bajo condiciones no controladas en Bogotá. Biomédica, 24 (4), 385-392.

Savage, H. \& Miller, B. House Mosquitoes of the U.S.A., Culex pipiens complex. Win Beats, 6, 8-9.

SIVIGILA. (2005). Semana epidemiológica. Sistema de Vigilancia en Salud Publica, $\mathrm{N}^{\circ} 43$, Instituto Nacional de Salud.

Thiery, I., Hamon, S., Delecluse, A. \& Orduz, S. (1998). The Introduction into Bacillus sphaericus of the Bacillus thuringiensis subsp. medellin Cyt1Ab1 Gene Results in Higher Susceptibility of Resistant Mosquito Larva Populations to
B. sphaericus. Appl Environ Microbiol, 64 (10), 3910-3916.

Travi, B. \& Montoya, J. Manual de entomología médica para investigadores de América Latina. Cali, Colombia: CIDEIM.

Vargas, M. (1998). El mosquito, un enemigo peligroso. Editorial Universidad de Costa Rica.

Villarreal, L. (1995). Bacillus sphaericus: para el control de vectores de enfermedades tropicales. Rev. Latinoam. Salud San. $A m b, 1,12-14$.

World Health Organization. (1985). Informal Consultation o the development of Bacillus sphaericus as a microbial larvicide. TDR/BCV/Sphaericus/. 85. $3 \mathrm{WHO/}$ $\mathrm{VBC} / 1-24$.

Yousten, A. \& Davidson, E. (1982). Ultra structural Analysis of spores and Parasporal Crystal formed by Bacillus sphaericus 2297. Applied and Environmental Microbiology, 44, 1449-1455.

Zahiri, N,S., Federici, B.A. \& Mulla, M.S. (2004). Laboratory and Simulated Field Evaluation of a New Recombinant of Bacillus thuringiensis ssp. israelensis and Bacillus sphaericus against Culex Mosquito Larvae (Diptera: Culicidae). J Med Entomol, 41 (3), 423-429. 